\title{
Index Graecitatis
}

\author{
$\dot{\alpha} \theta \alpha u \mu \alpha \sigma \tau i ́ \alpha=$ assenza di meraviglia

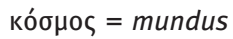

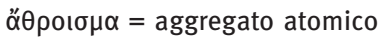

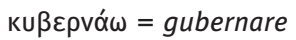

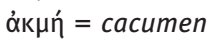 \\ 入óyos = ratio \\ $\dot{\alpha} \mu \alpha \rho i^{\prime} \alpha=$ culpa

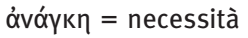 \\ $\alpha \dot{\alpha} \alpha \theta u \mu i ́ \alpha \sigma ı \varsigma$ = esalazione

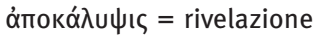

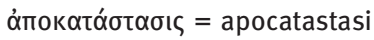

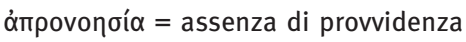 \\ $\lambda$ 入ıнó $\varsigma$ = pestilenza \\ $\mu \alpha \kappa \alpha$ роótn $=$ beatitudine \\ $\mu \alpha \kappa \rho \alpha ́ v \theta \rho \omega \pi о \varsigma=$ corpo cosmico \\ $\mu \varepsilon \gamma \alpha \lambda \circ \psi u x i ́ \alpha=$ magnanimità \\ $\mu \varepsilon \tau \alpha \beta o \lambda \eta ́$ = trasformazione

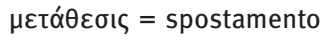 \\ $\alpha \dot{\alpha} \alpha \rho \alpha \xi \dot{\prime} \alpha=$ assenza di turbamento

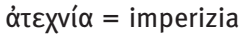

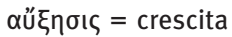

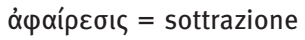

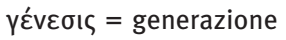 \\ $\gamma \tilde{n} \rho \alpha \varsigma=$ vetustas

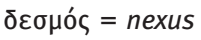 \\ $\Delta \eta \mu \mathrm{Ioupyó} \varsigma=$ Demiurgo \\ $\delta ı \alpha$ кó $\mu \eta \sigma \iota \varsigma=$ diacosmesi

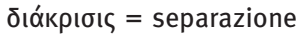

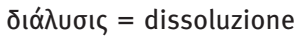 \\ $\delta ı \alpha \mu v \eta^{\prime}=$ permanenza del cosmo \\ סĩvo $\varsigma=$ vortice \\ ঠó $\alpha$ = opinione

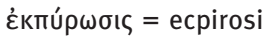

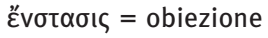 \\ ह̌ $\xi ı \varsigma$ = forza coesiva

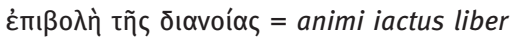 \\ $\mu \varepsilon \tau \alpha \kappa o ́ \sigma \mu \iota \alpha=$ intermondi \\ $\mu \varepsilon \tau \varepsilon \dot{\varepsilon} \omega \rho \alpha$, $\tau \grave{\alpha}=$ fenomeni celesti \\ $\mu \tilde{\theta} \theta 0 \varsigma=$ mito \\ Nعĩко $=$ Discordia

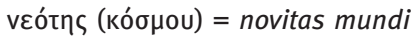 \\ vóбoৎ = malattia \\ $\pi \tilde{\alpha} v$ (tò) = summarum summa \\ $\pi \alpha \rho \alpha \dot{\lambda} \lambda \alpha \xi_{\llcorner\varsigma}=$ deviazione astrale

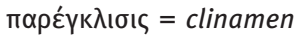

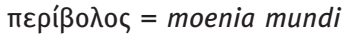

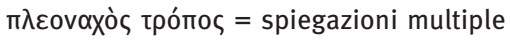 \\ бúүкрıбıৎ = aggregazione

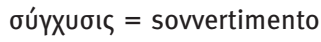 \\ $\sigma$ ó $\sigma$ tๆ $\mu \alpha=$ costruzione

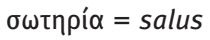 \\ üßeıs = tracotanza \\ ǔ $\psi \circ \varsigma=$ sublime

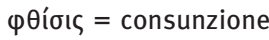 \\ ทंठoví = voluptas \\ $\theta \alpha \tilde{u} \mu \alpha=$ mirum \\ $\varphi \theta$ ○ó $=$ ruina \\ $\Phi ı \lambda i ́ \alpha=$ Amore \\ $\theta \varepsilon \mu \varepsilon \dot{\lambda} \lambda \alpha=$ fondamenta

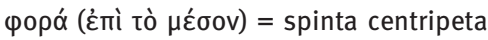

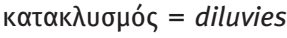 \\ Xóos $=$ Caos
}

кєvóv (tò) = inane 